\title{
Investigation of Periodic-Disturbance Identification and Rejection in Spacecraft
}

\author{
Jimmy Lau* and Sanjay S. Joshi ${ }^{\dagger}$ \\ University of California, Davis, California 95616 \\ and \\ Brij N. Agrawal ${ }^{\ddagger}$ and Jong-Woo Kim ${ }^{\S}$ \\ Naval Postgraduate School, Monterey, California 93943
}

\begin{abstract}
Spacecraft periodic-disturbance rejection using a realistic spacecraft hardware simulator and its associated models is investigated. The effectiveness of the dipole-type disturbance rejection filter on the current realistic nonlinear rigid-body spacecraft model is validated. However, it is shown that the rejection filter is not robust to disturbance frequency uncertainty. Therefore, system identification techniques are needed if the disturbance frequency is unknown or changing. Based on experience with the current test bed, two simple closed-loop system identification methods are introduced to experimentally identify the disturbance frequency using data from the control input torque signal. It is shown that an incorrect filter frequency results in beating of the control input torque. Examination of the beat envelope leads to tuning of the rejection filter.
\end{abstract}

\section{Introduction}

A COMMON challenge in high-performance spacecraft control is the mitigation of periodic disturbances caused by internal or external components. Examples include disturbances caused by control moment gyroscopes (CMGs) ${ }^{1}$ and disturbances caused by the excitation of flexible appendages, as was the case with the Hubble Space Telescope. ${ }^{2}$ In the present paper, disturbance-rejection filters are studied using the Naval Postgraduate School (NPS) Three-Axis Simulator (TAS) test bed. The TAS was developed at the NPS in Monterey, California and is shown in Fig. 1. The purpose of the TAS was to prove a satellite's ability to redirect a laser originating from Earth, an aircraft, or another satellite to another location on Earth for communication or defense applications. A satellite utilizing lasers requires exceptional acquisition, pointing, and tracking capabilities. On-going research has been conducted to study fine acquisition, pointing, and tracking requirements. ${ }^{3,4}$ Periodic disturbances can be introduced into the test bed by deflecting a flexible beam on the test bed.

Disturbance rejection of periodic disturbances is a classical field that has been examined using a variety of methods, including both feedforward and feedback methods. The current paper further examines the dipole disturbance-rejection filter (DRF), which explicitly employs the periodic-disturbance frequency into a filter embedded in the feedback control loop. The DRF is based on the internal model principle, ${ }^{5-7}$ and its attenuation effectiveness has been shown in a simulation of the Hubble Space Telescope ${ }^{8}$ and in simulations for a spacecraft with CMGs. ${ }^{1,9}$ The filter's effectiveness was also shown experimentally on NASA's minimast truss structure at the NASA Langley Research Center ${ }^{10}$ and on NASA's Advanced Control Evaluation for Structures test bed at the NASA Marshall Space Flight

Received 22 April 2005; revision received 8 September 2005; accepted for publication 13 September 2005. Copyright (C) 2005 by Sanjay S. Joshi. Published by the American Institute of Aeronautics and Astronautics, Inc., with permission. Copies of this paper may be made for personal or internal use, on condition that the copier pay the $\$ 10.00$ per-copy fee to the Copyright Clearance Center, Inc., 222 Rosewood Drive, Danvers, MA 01923; include the code 0731-5090/06 \$10.00 in correspondence with the CCC.

${ }^{*}$ Graduate Research Assistant, Department of Mechanical and Aeronautical Engineering.

${ }^{\dagger}$ Assistant Professor, Department of Mechanical and Aeronautical Engineering, Bainer Hall, 1 Shields Avenue; maejoshi@ucdavis.edu. Senior Member AIAA.

\$Distinguished Professor and Director, Spacecraft Research and Design Center. Associate Fellow AIAA.

${ }^{\S}$ Postdoctoral Research Fellow, Spacecraft Research and Design Center.
Center. ${ }^{11}$ The Hubble Space Telescope solar array disturbance problem was also attenuated by the same filter, although the filter was not termed the dipole DRF. ${ }^{2}$

The dipole DRF is based on linear theory. Therefore, it is of interest to examine its efficacy on the current nonlinear spacecraft model. In addition, the robustness of the rejection filter is explored in the current paper. It is shown that the proposed rejection filter is not robust to frequency uncertainty. Based on experience with the TAS, simple closed-loop system identification methods are introduced to identify experimentally the disturbance frequency to be used within the filter design.

The paper is organized as follows. In Sec. II, the theory behind the DRF is reviewed. In Sec. III, the hardware test bed and associated linear and nonlinear system models are described. In Sec. IV, the DRF is demonstrated on the test bed models. In Sec. V, robustness of the filter to frequency uncertainty is investigated. As expected, the DRF's attenuation effectiveness is based on accurate knowledge of the disturbance frequency. In Sec. VI, motivated by test bed results, two methods are introduced to determine experimentally the disturbance frequency when using DRFs, and the advantages of each are discussed. Finally, in Sec. VII, the paper is concluded with a summary of results and future research directions.

\section{Dipole DRF}

The dipole DRF is based on the internal model principle, which places periodic-disturbance poles inside the compensator loop so that the unstable disturbance poles are canceled during loop closure between the output signal and input disturbance (Fig. 2). ${ }^{5}$ Equation (1) shows the closed-loop transfer function between the input disturbance signal $G_{\text {dist }}(s)$ and output signal $Y(s)$ of Fig. 2. In closed loop, the disturbance poles are canceled by the inclusion of a DRF,

$$
\begin{aligned}
Y(s) & =\frac{1 / D(s)}{1+N(s) N_{c}(s) / D(s) D_{c}(s) D_{\text {dist }}(s)} G_{\text {dist }}(s) \\
& =\left[\frac{D_{c}(s) D_{\text {dist }}(s)}{D_{c}(s) D_{\text {dist }}(s) D(s)+N_{c}(s) N(s)}\right] \frac{N_{\text {dist }}(s)}{D_{\text {dist }}(s)}
\end{aligned}
$$

Although the traditional DRF shown in Fig. 2 does not include a numerator, a numerator with the same order as the denominator should be chosen to ensure a zero for every pole in the DRF root locus. ${ }^{6,7}$ To derive the DRF, first consider the Laplace transform of a persistent periodic disturbance of amplitude $A$ and a single 


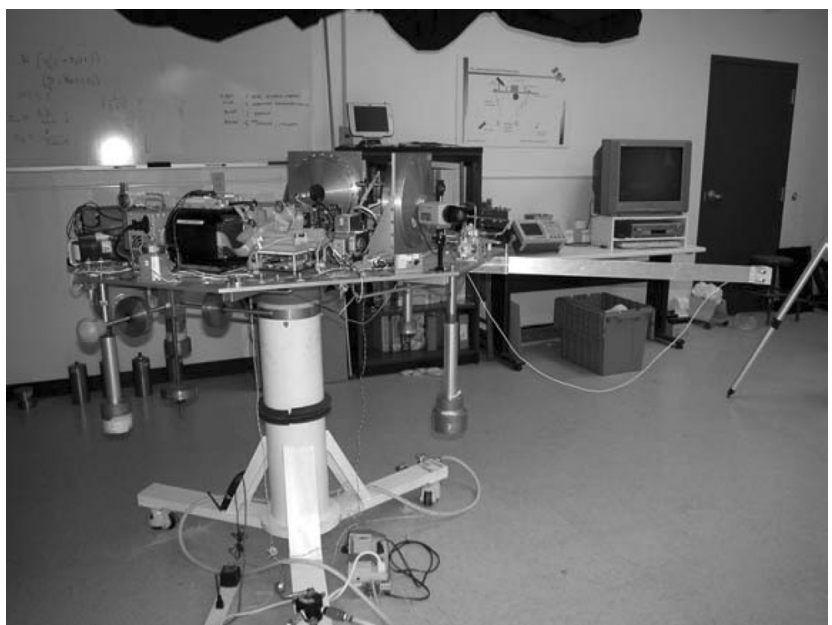

Fig. 1 NPS's Three-Axis Simulator test bed.

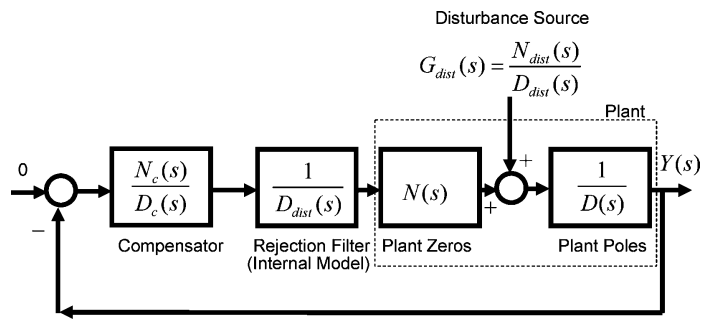

Fig. 2 Closed-loop disturbance rejection control system based on internal model principle.

frequency $\omega$ that can be represented as

$$
G_{\mathrm{dist}}(s)=\left[\frac{A / \omega}{s^{2} / \omega^{2}+1}\right]
$$

Only the disturbance denominator (pole) is of interest. Therefore, in the filter design, the persistent periodic-disturbance frequency $\omega$ is employed as the disturbance rejection frequency $\omega_{p}$, and the DRF becomes

$$
G_{\mathrm{DRF}}(s)=\frac{s^{2} / \omega_{z}^{2}+1}{s^{2} / \omega_{p}^{2}+1}
$$

Note that the DRF numerator is included as a modification of Eq. (2), and recall that the numerator and denominator should be of the same order. The DRF Bode plot is shown in Fig. 3, and some guidelines in selecting $\omega_{z}$ are provided in the next paragraph.

An $\omega_{p}$ and $\omega_{z}$ pair is termed a dipole, and the difference between the $\omega_{p}$ and $\omega_{z}$ values is termed the dipole strength. ${ }^{7}$ The settling time of the transient response increases as the difference between the dipole terms increases. The $\omega_{z}$ can be chosen to be less than or greater than $\omega_{p}$ as shown in Fig. 3. A magnitude magnification in the high frequencies results from $\omega_{z}>\omega_{p}$, and a magnitude attenuation in the high frequencies results from $\omega_{z}<\omega_{p}$. The net magnitude attenuation or magnification increases as the dipole strength increases. It is suggested that $\omega_{z}$ be selected to be between two consecutive poles of the system for stability. ${ }^{6}$

Shown in Eq. (3), the DRF is basically a second-order generalized filter ${ }^{7} G_{c}(s)$ [Eq. (4)], without damping terms. Actually, damping terms can be included as reported in the literature. ${ }^{8}$ In a Hubble Space Telescope solar array disturbance analysis, the numerator damping term was made high compared to the denominator damping term, which made the antiresonance practically nonexistent. ${ }^{8}$ The filter was designed by adjusting the damping terms to create a second-order non-minimum phase bandpass filter. Note that for disturbances of several frequencies, dipoles may be included for each
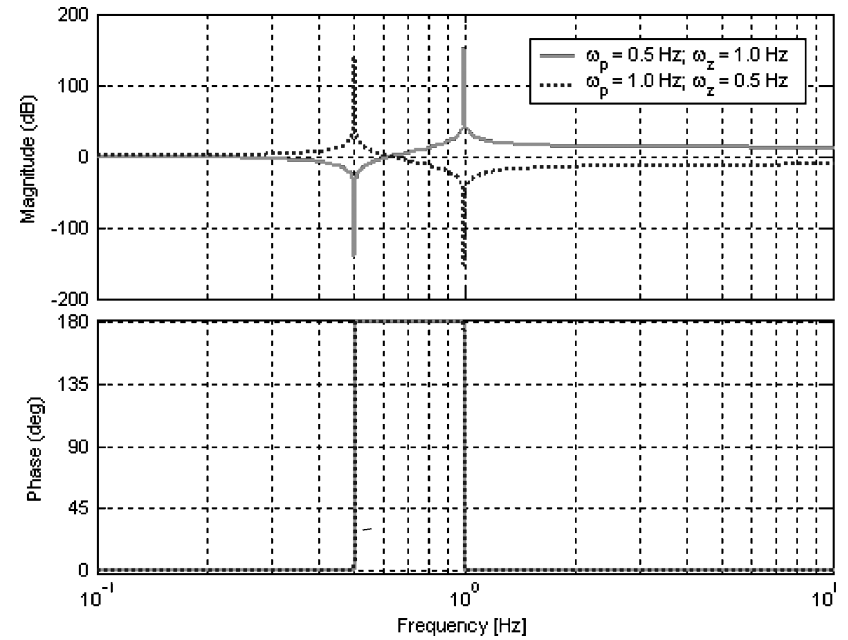

Fig. 3 DRF Bode diagram.

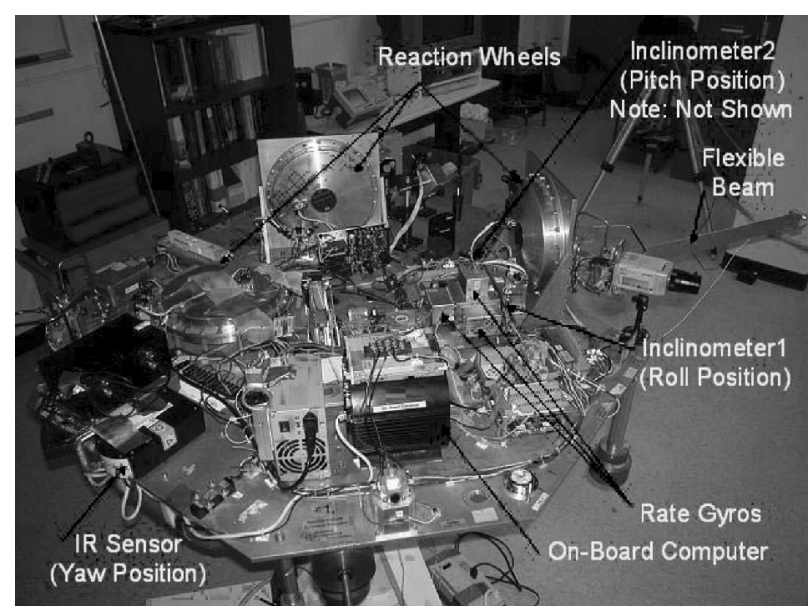

Fig. 4 TAS components.

frequency, ${ }^{6}$

$$
G_{c}(s)=\frac{s^{2} / \omega_{z}^{2}+\left(2 \zeta_{z} / \omega_{z}\right) s+1}{s^{2} / \omega_{p}^{2}+\left(2 \zeta_{p} / \omega_{p}\right) s+1}
$$

\section{NPS's TAS and Its Linear and Nonlinear Simulation Models}

The DRF and the underlying internal model principle are all based on linear system theory. Studies of DRF filters on linear system models of spacecraft have been reported, $, 6,7,12$ and results validate the linear system theory as expected. In this paper, the DRF is only demonstrated on a nonlinear model of the experimental test bed. However, the proposed input disturbance frequency determination methods discussed in Sec. VI are analyzed using both a linear and nonlinear simulation model.

The TAS hardware simulator's components are shown in Fig. 4. The simulator underside has a semispherical ball that mates with an air bearing's semispherical cup. The TAS essentially has threeaxis frictionless motion when pressure is supplied through the air bearing, which produces a thin film of air between the semispherical ball and cup.

Removable hanging masses and fine-tuning masses are utilized to balance the TAS such that the center of mass corresponds to the TAS center of rotation. In doing so, the TAS emulates a satellite in a space environment. However, perfect balancing cannot be realistically achieved by manual tuning, and so a control input torque must continually counter the imbalance disturbance torque.

For actuators, the TAS has three reaction wheels that are orthogonal to each other. The TAS uses two inclinometers and a oneaxis infrared (IR) sensor for attitude sensing. The inclinometers are 


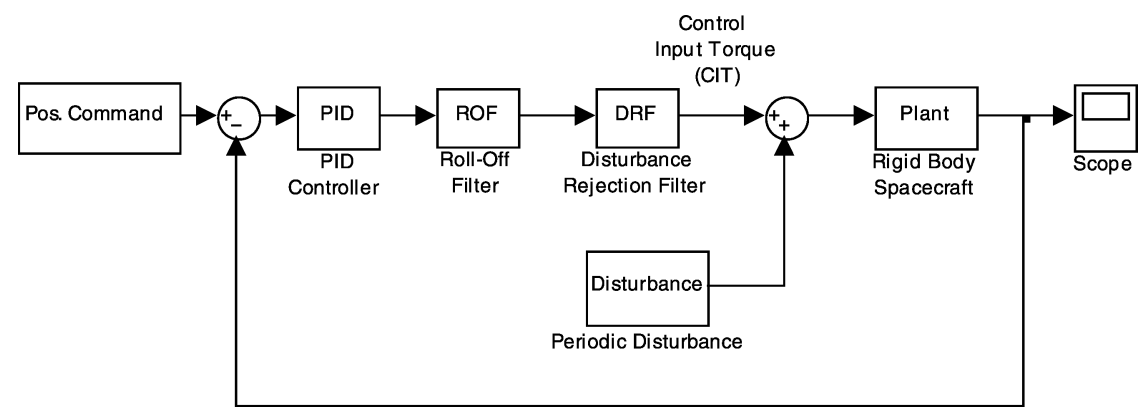

Fig. 5 Closed-loop block diagram of rigid-body spacecraft with PID controller, ROF, and DRF.

capacitive liquid-based sensors where the sensors' capacitance is a linear function of the liquid angle. Although inclinometers are not used on actual spacecraft, inclinometers provide a reasonably accurate and inexpensive alternative to determine the TAS pitch and roll angles. The IR sensor has two phototransistors that measure the change in intensity of a light source to determine the TAS yaw angle. For angular rate, the TAS uses three orthogonal mechanical rate gyros. The TAS uses MATLAB/Simulink ${ }^{\mathrm{TM}}$ and $\mathrm{xPC}^{\text {Target }}{ }^{\mathrm{TM}}$ for real-time control. More details of the hardware design may be found in Ref. 12.

The TAS is physically subjected to a periodic disturbance produced from the initial displacement of an aluminum point mass and beam that is attached to the edge of the TAS as shown in Fig. 1. The disturbance parameters were experimentally derived ${ }^{12}$ as $A=2.13 \mathrm{~N} \cdot \mathrm{m}$ (initial disturbance amplitude), $\omega_{n}=0.61512 \mathrm{~Hz}$ (natural frequency), and $\xi=0.002303$ (damping ratio). Note that in the current study, the beam is considered only as a source of disturbance to rigid-body models.

\section{A. TAS Single-Axis Linear Simulation Model}

The single-axis rigid-body linear TAS simulation block diagram is shown in Fig. 5. The simulation consists of a proportional integral derivative (PID) controller, roll-off filter (ROF), DRF, disturbance signal, and a rigid-body spacecraft plant. The transfer function for the PID controller, ROF, and plant are shown in Eqs. (5-7). The disturbance signal is represented in Eq. (2). Similarly, the DRF is represented in Eq. (3),

$$
\begin{aligned}
& G_{\mathrm{PID}}(s)=\left\{\left(\tau_{d} k / s\right)\left[s^{2}+\left(1 / \tau_{d}\right) s+\left(1 / \tau_{d} \tau_{i}\right)\right]\right\} \\
& =\{[14.4686(1.1871) / s][s+2 \pi(0.005)][s+2 \pi(0.006)]\}
\end{aligned}
$$

where $k, \tau_{i}$, and $\tau_{d}$ are the proportional, integral, and derivative (PID) gains, respectively.

$$
\begin{gathered}
G_{\mathrm{ROF}}(s)=\{2 \pi(0.9) /[s+2 \pi(0.9)]\} \\
G_{p}(s)=\left[1 / J s^{2}\right]=\left[1 / 55 s^{2}\right]
\end{gathered}
$$

where $J\left(\mathrm{~kg} \cdot \mathrm{m}^{2}\right)$ is the rigid-body inertia about the single axis.

The PID controller and ROF were designed by classical frequency response shaping methods to maximize the gain and phase margins and were validated on the hardware test bed. ${ }^{12}$ The inertia parameter shown in Eq. (7) was experimentally determined. ${ }^{12}$

\section{B. TAS Three-Axis Nonlinear Rigid-Body Simulation Model}

The three-axis nonlinear rigid-body TAS simulation is based on Euler's rotational equation and the standard kinematic differential equation for quaternions, which are shown in Eqs. (8) and (10), respectively. $7,13,14$ Note that in our model, the roll and pitch axes (axes 1 and 3) are aligned from the central point of the test bed table through the centerlines of the pitch and roll reaction wheels shown in Fig. 4. The yaw axis (axis 2) is aligned with the vertical air bearing support structure shown in Fig. 1.

$$
\frac{{ }^{B} \mathrm{~d}}{\mathrm{~d} t}\left(J \boldsymbol{\omega}^{B / N}\right)+\boldsymbol{\omega}^{B / N} \times J \boldsymbol{\omega}^{B / N}={ }^{B} \boldsymbol{u}_{\text {control }}+{ }^{B} \boldsymbol{T}_{\mathrm{dist}}+{ }^{B} \boldsymbol{T}_{\mathrm{imb}}
$$

where

$$
\begin{gathered}
\boldsymbol{J}=\left[\begin{array}{ccc}
31.8 & 5 & 1 \\
5 & 55 & 3 \\
1 & 3 & 31.8
\end{array}\right] \mathrm{kg} \cdot \mathrm{m}^{2}, \quad{ }^{B} \boldsymbol{u}_{\text {control }}=\left[\begin{array}{l}
u_{1} \\
u_{2} \\
u_{3}
\end{array}\right] \mathrm{N} \cdot \mathrm{m} \\
{ }^{B} \boldsymbol{T}_{\text {dist }}=\left[\begin{array}{c}
0 \\
2.1313 \sin (3.8648 t) \\
0
\end{array}\right] \mathrm{N} \cdot \mathrm{m}, \quad{ }^{B} \boldsymbol{T}_{\mathrm{imb}}=\left[\begin{array}{l}
\mathrm{imb}_{1} \\
\mathrm{imb}_{2} \\
\mathrm{imb}_{3}
\end{array}\right] \mathrm{N} \cdot \mathrm{m}
\end{gathered}
$$

Figure 6 shows the three-axis nonlinear rigid-body TAS Simulink model used to analyze the dipole filter. An attitude command is prescribed that is then transformed to a rate command using Eq. (9), assuming a 2-3-1 rotation sequence. ${ }^{7}$

$$
\begin{aligned}
{\left[\begin{array}{c}
\dot{\theta}_{1} \\
\dot{\theta}_{2} \\
\dot{\theta}_{3}
\end{array}\right] } & =\frac{1}{\cos \theta_{3}}\left[\begin{array}{ccc}
\cos \theta_{3} & -\cos \theta_{1} \sin \theta_{3} & \sin \theta_{1} \sin \theta_{3} \\
0 & \cos \theta_{1} & -\sin \theta_{1} \\
0 & \sin \theta_{1} \cos \theta_{3} & \cos \theta_{1} \cos \theta_{3}
\end{array}\right] \\
& \times\left[\begin{array}{l}
\omega_{1}^{\text {Command }} \\
\omega_{2}^{\text {Command }} \\
\omega_{3}^{\text {Command }}
\end{array}\right]
\end{aligned}
$$

The measured or actual rate is solved by Euler's rotational equation, which is shown in Eq. (8). The rate is then fed back so that a rate error signal is calculated by taking the difference between the commanded and actual rate. At the same time, the actual rate is used in Eq. (10) to calculate the actual attitude in quaternions, 7,14

$$
\left[\begin{array}{l}
\dot{q}_{1} \\
\dot{q}_{2} \\
\dot{q}_{3} \\
\dot{q}_{4}
\end{array}\right]=\frac{1}{2}\left[\begin{array}{cccc}
0 & \omega_{3} & -\omega_{2} & \omega_{1} \\
-\omega_{3} & 0 & \omega_{1} & \omega_{2} \\
\omega_{2} & -\omega_{1} & 0 & \omega_{3} \\
-\omega_{1} & -\omega_{2} & -\omega_{3} & 0
\end{array}\right]\left[\begin{array}{l}
q_{1} \\
q_{2} \\
q_{3} \\
q_{4}
\end{array}\right]
$$

where $\vec{q}=\left[\begin{array}{llll}q_{1} & q_{2} & q_{3} & q_{4}\end{array}\right]^{T}, \quad \overrightarrow{\dot{q}}=\left[\begin{array}{llll}\dot{q}_{1} & \dot{q}_{2} & \dot{q}_{3} & \dot{q}_{4}\end{array}\right]^{T}$, and $\boldsymbol{\omega}^{B / N}=$ $\left[\begin{array}{ccc}\omega_{1} & \omega_{2} & \omega_{3}\end{array}\right]^{T}$.

The quaternion vector is transformed to Euler angles where the actual attitude is fed back to the commanded attitude so that the attitude error signal is calculated. The conversion from quaternions to Euler angles for a 2-3-1 rotational sequence is shown in Eqs. (1113) (Ref. 7). The attitude and rate error signals are utilized in the PID controller. A control input torque (CIT) is generated after the DRF (Fig. 6).

$$
\begin{gathered}
\theta_{2}=\tan ^{-1}\left[-\frac{2\left(q_{1} q_{3}-q_{2} q_{4}\right)}{1-2\left(q_{2}^{2}+q_{3}^{2}\right)}\right] \\
\theta_{3}=\sin ^{-1}\left[2\left(q_{1} q_{2}+q_{3} q_{4}\right)\right] \\
\theta_{1}=\tan ^{-1}\left[-\frac{2\left(q_{3} q_{2}-q_{1} q_{4}\right)}{1-2\left(q_{1}^{2}+q_{3}^{2}\right)}\right]
\end{gathered}
$$

The total external torque shown on the right-hand side of Eq. (8) includes the CIT, TAS imbalance disturbance torque, and a periodic 


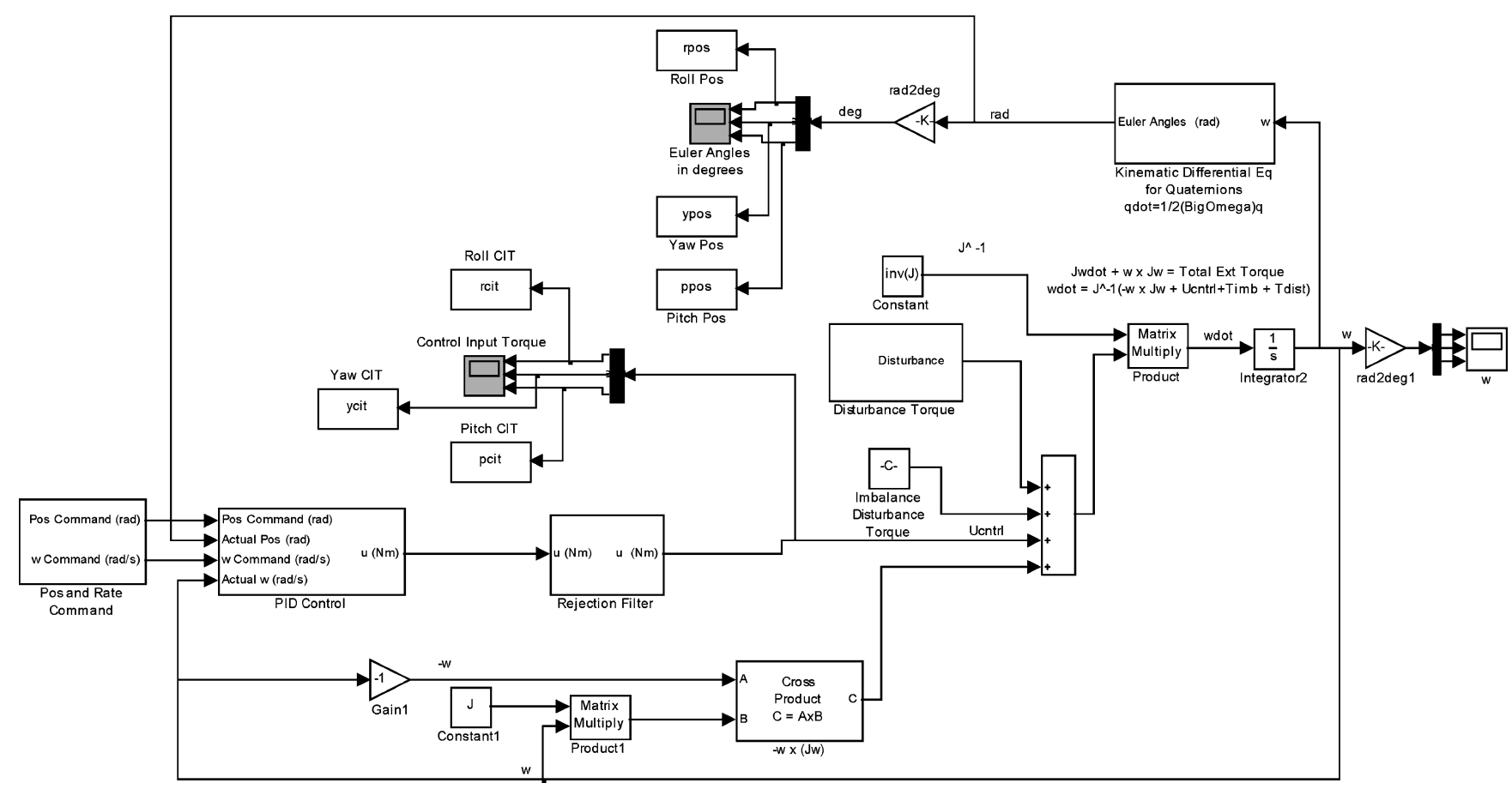

Fig. 6 Three-axis nonlinear rigid-body TAS simulation model.

disturbance torque about the yaw axis. All of the torques are in the spacecraft body frame (B) and are represented in the Simulink model shown in Fig. 6. Again, note that flexibility in the beam is not considered in the current model.

The TAS imbalance disturbance torques in Eq. (8) were approximated using the hardware test bed's CIT experimental data and each are on the order of $0.1 \mathrm{~N} \cdot \mathrm{m}$. The imbalance torque is the result of the center of mass not coinciding with the center of rotation. This effect changes slightly depending on the success of each balancing of the test bed. A bias in the CIT experimental data approximates the magnitude of imbalance being countered, shown later. For the inertia matrix shown in Eq. (8), the inertia about the yaw axis was experimentally determined. ${ }^{12}$ The inertia about the pitch and roll axes were analytically determined as described in Ref. 12. The products of inertia were assumed small compared to the diagonal inertias. Small values were estimated so that coupling between the axes would also be small, based on the assumption that our coordinate system is close to the principal axes. To verify numerically the final threeaxis simulation (3-AS), the sum of all torques in the zero command case was verified as zero as expected from theory. ${ }^{12}$

\section{Effect of DRF on TAS Nonlinear Model}

The DRF used in the simulation experiments is expressed as shown in Eq. (14). The $\omega_{z}$ selected follows the guideline of being between two consecutive poles of the system. ${ }^{6}$ The $\omega_{z}$ is placed between one of the plant poles at the origin and the DRF's pole,

$$
G_{\mathrm{DRF}}(s)=\left[\frac{s^{2} / \omega_{z}^{2}+1}{s^{2} / \omega_{p}^{2}+1}\right]=\frac{0.1013 s^{2}+1}{0.06695 s^{2}+1}
$$

Figures 7 and 8 show the DRF's effectiveness on a persistent periodic input disturbance on the yaw. The attitude command is a 1-deg position command in the yaw and a 0 -deg position command in the pitch and roll to emulate the TAS stabilization process. The DRF is extremely effective in reducing the effect of the disturbance on the yaw position.

Note that we were not able to implement the DRF on the hardware test bed as a result of actuator saturation. Our reaction wheel actuators were limited to $0.2 \mathrm{~N} \cdot \mathrm{m}$, whereas our required torque was $2.13 \mathrm{~N} \cdot \mathrm{m}$ (to duplicate the simulation results). A $0.2-\mathrm{N} \cdot \mathrm{m}$ amplitude disturbance produces inconsequential effects of the test bed yaw position, due to the comparatively large inertia of the test bed.

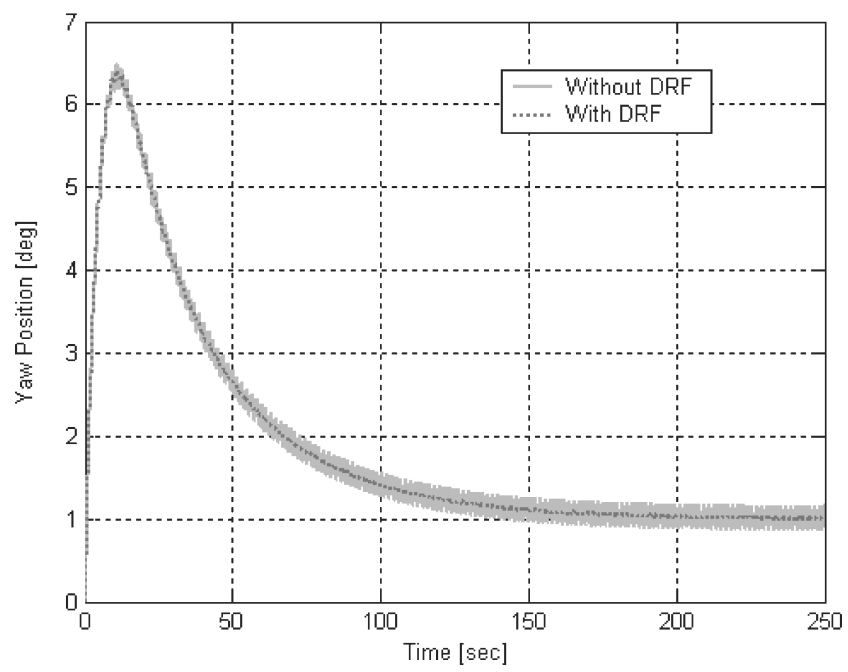

Fig. 7 DRF effect on persistent periodic disturbance of $0.6151 \mathrm{~Hz}$ and $2.13 \mathrm{~N} \cdot \mathrm{m}$ peak magnitude in 3-axis non-linear simulation.

\section{DRF Robustness to Frequency Uncertainty}

Figure 7 shows the DRF capability in attenuating periodic input disturbances. However, the results are based on the assumption that the designer knows exactly the disturbance frequency. In Fig. 8 the response when the DRF is designed to reject the correct and several incorrect input disturbance frequencies is compared. Note that the DRF is not very robust because there is negligible attenuation when the DRF rejection frequency $\left[\omega_{p}\right.$ in Eq. (3)] is incorrect by $\pm 0.033 \mathrm{~Hz}$ (approximately $5 \%$ frequency uncertainty). In the next section, we will describe two simple system identification methods to determine explicitly the filter design frequency.

\section{Input Disturbance Frequency Determination}

It was shown in the 3-AS that the DRF is not robust to frequency uncertainty. If the input disturbance frequency is uncertain or changing, the DRF's attenuation may be negligible. Therefore, the ability to determine the input disturbance frequency is essential for the designer. In this section, two closed-loop methods to determine the input disturbance frequency will be shown, based on experience 


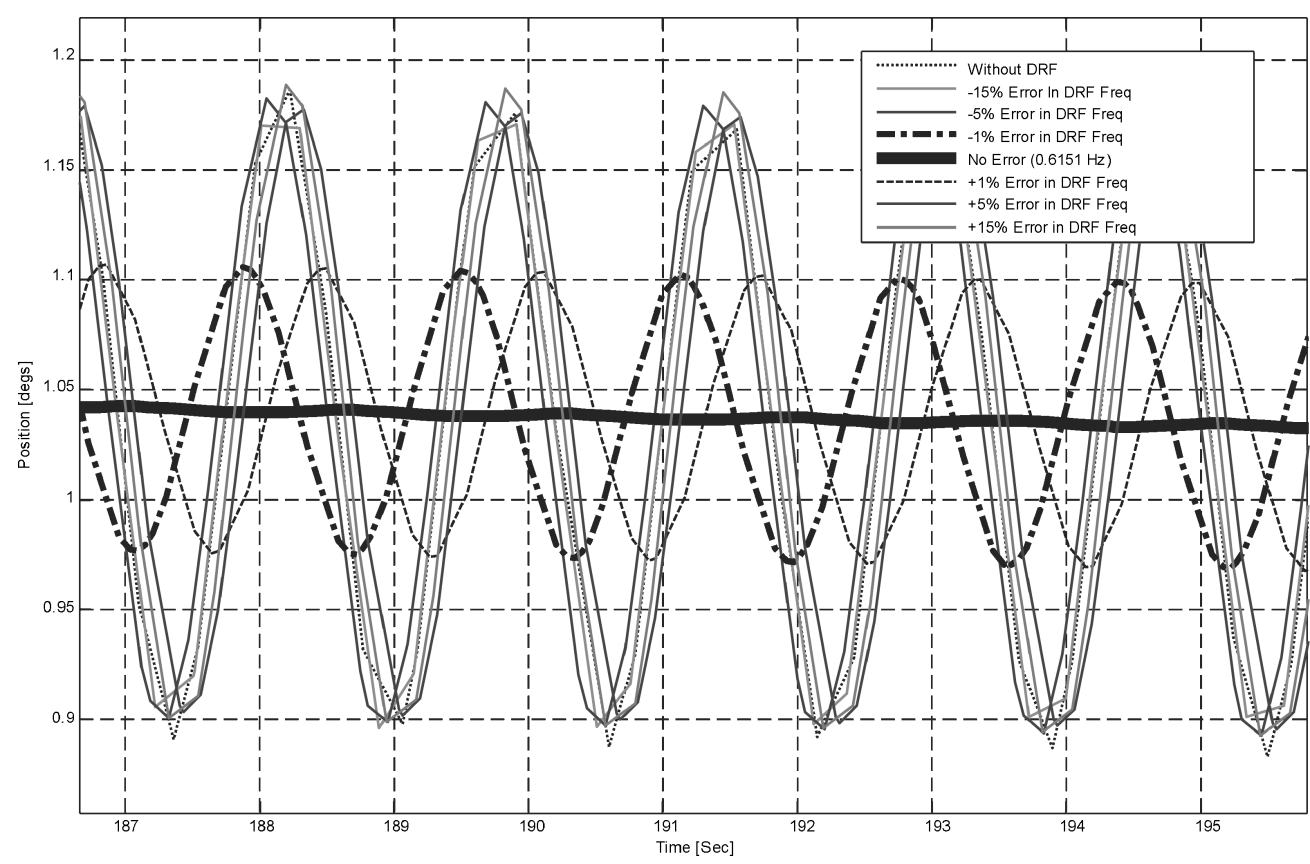

Fig. 8 Effects of correct and incorrect DRF rejection frequency where persistent periodic disturbance is 0.6151 Hz in 3-AS (same conditions as in Fig. 7).
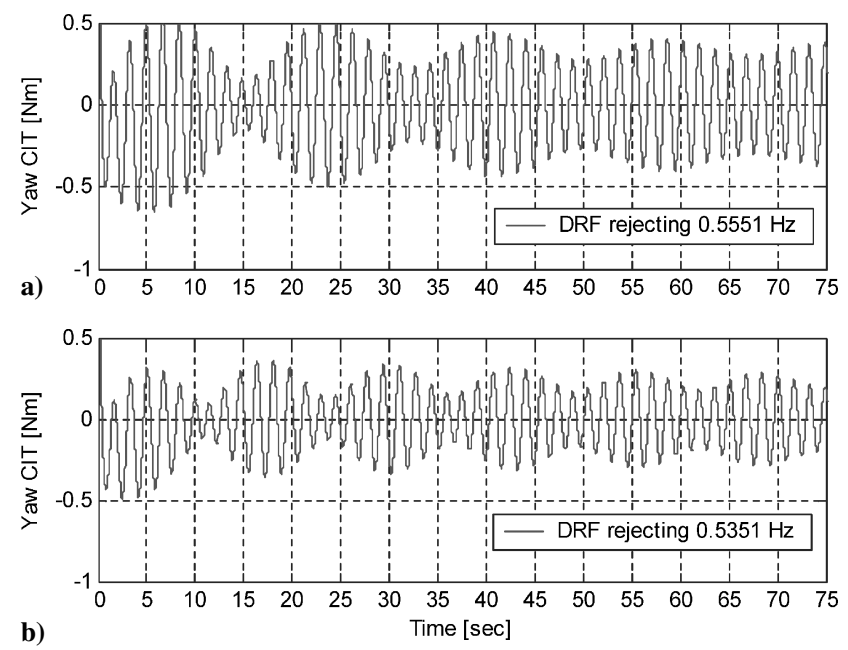

Fig. 9 Yaw CIT when DRF rejection frequency is not equivalent to disturbance frequency in single-axis linear simulation (persistent disturbance frequency $=0.6151 \mathrm{~Hz}$ ).

with the TAS. The first method evaluates the CIT plot and simply counts the number of cycles in a time range. The second method exploits a beating phenomenon in the CIT that results from an inaccurate filter design frequency. Both methods will be demonstrated in linear and nonlinear simulations, as well as in hardware experiments.

Figure 9 shows the CIT plot from the single-axis linear simulation (Fig. 5). Figure 9a shows the CIT in closed-loop from the linear model when the DRF is designed using a $0.5551-\mathrm{Hz}$ disturbance rejection frequency $\left[\omega_{p}\right.$ in Eq. (3)], and Fig. 9b shows the CIT in closed-loop when the DRF is designed using a $0.5351-\mathrm{Hz}$ disturbance rejection frequency $\omega_{p}$, where the actual input disturbance frequency for both is $0.6151 \mathrm{~Hz}$. For Figs. $9 \mathrm{a}$ and $9 \mathrm{~b}, \omega_{z}$ is $0.5 \mathrm{~Hz}$ [Eq. (3)].

For the first frequency determination method, the input disturbance frequency is found by measuring the high-frequency oscillation in Fig. 9. This high-frequency oscillation in Figs. 9a and 9b both reveal the input disturbance frequency of $0.6151 \mathrm{~Hz}$.

Shown in Fig. 9, note that a beating phenomenon is occurring, the result of a superposition of two waves with slightly differ-
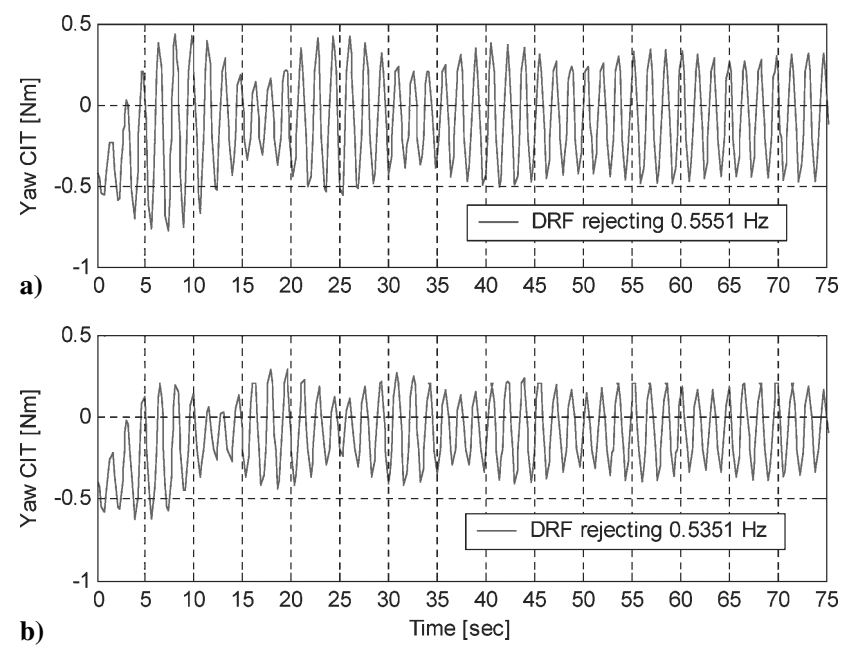

Fig. 10 Yaw CIT when DRF rejection frequency is not equivalent to disturbance frequency in 3-axis non-linear simulation (persistent disturbance frequency $=0.6151 \mathrm{~Hz}$ ).

ing frequencies. ${ }^{15}$ Through linear and nonlinear simulation studies and hardware test bed experiments, it was found that if the filter design frequencies obey the relation to the actual disturbance frequencies as $\omega_{z}<\omega_{p}<$ actual-disturbance-frequency in Eq. (3), then beats appear in the Fig. 9 plot. For Fig. 9, recall that $\omega_{p}$ is $0.5551 \mathrm{~Hz}$ and $0.5351 \mathrm{~Hz}$ for Figs. 9a and 9b, respectively. Figure $9 \mathrm{~b}$ shows more beats than Fig. 9a and also shows a decrease in CIT. Therefore, as $\omega_{p}$ approaches $\omega_{z}$, which is $0.5 \mathrm{~Hz}$, more beats occur in the CIT and the CIT decreases. If $\omega_{p}$ reached $\omega_{z}$, then Eq. (3) would equate to 1.0 as if the rejection filter were not used. As $\omega_{p}$ approaches the actual input disturbance frequency, less beats occur, and the CIT increases to approach the disturbance amplitude. $^{12}$

To validate the beating phenomenon, disturbances and filters were investigated in both the three-axis nonlinear simulation model and on the actual hardware test bed. The CIT plot from the nonlinear simulation using the same disturbance and filter conditions of Fig. 9 is shown in Fig. 10. Note that beating is once again present. 

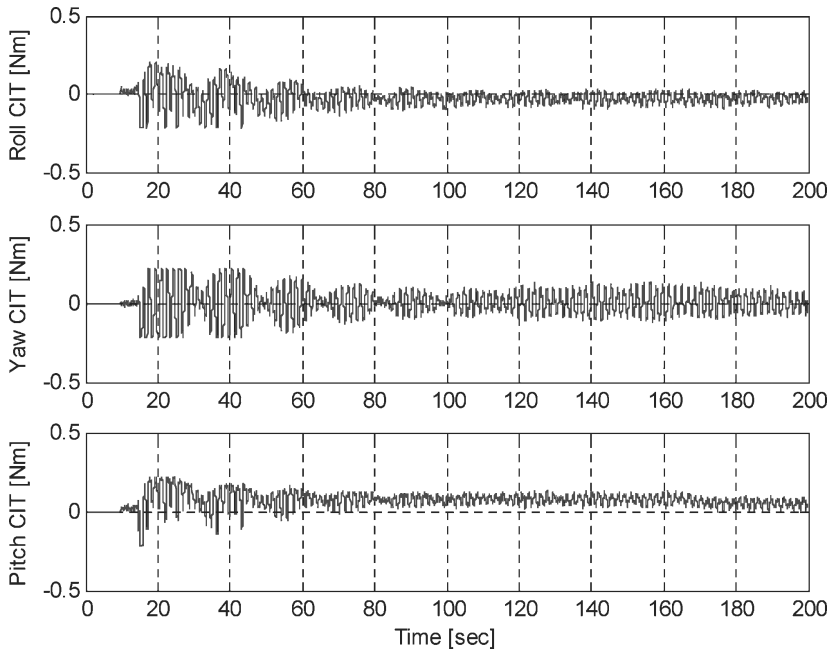

Fig. 11 Experimental CIT responses for 0.6151-Hz decaying periodic disturbance when DRF rejection frequency is $0.5551 \mathrm{~Hz}$; note that pitch CIT is biased due to imbalance of test bed.

The same phenomenon was also experimentally validated on the hardware TAS. The hardware TAS parameters are equivalent to the simulation parameters (which were originally derived from the TAS testbed). Hereafter the TAS attempts to maintain an Euler angle attitude of $\left[\begin{array}{lll}0 & 0 & 0\end{array}\right]^{T}$ while experiencing a decaying (due to beam damping) periodic input disturbance. The disturbance frequency is $0.6151 \mathrm{~Hz}$ and the DRF rejection frequency is $0.5551 \mathrm{~Hz}$. The resulting CIT response is shown in Fig. 11. Again, beats are present.

A frequency determination method employing a DRF and CIT beats will now be shown using the nonlinear simulation results (Fig. 10). Note that the method could have been demonstrated with either the linear simulation (Fig. 9) or the hardware experiment results (Fig. 11). The CIT beat frequency $f_{b}$, actual input disturbance frequency $f_{\text {dist }}$, and DRF rejection design frequency $f_{\text {DRF }}$, are known to be related by ${ }^{15}$

$$
f_{b}=f_{\text {dist }}-f_{\mathrm{DRF}}
$$

Note that Eq. (15) is simply describing the definition of a beat frequency. Therefore, in this case, the beat frequency is produced by the slightly differing frequencies of the actual input disturbance frequency and the filter rejection frequency. In Fig. 10a, the beat frequency (hertz) is shown to be

$$
f_{b 1}=[1.5 /(50-25)][\text { beats } / \mathrm{s}]=0.06
$$

and in Fig. 10b the beat frequency (hertz) is

$$
f_{b 2}=[2 /(37.5-12.5)][\text { beats } / \mathrm{s}]=0.08
$$

where $f_{\mathrm{DRF} 1}$ is $0.5551 \mathrm{~Hz}$ for Fig. $10 \mathrm{a}$ and $f_{\mathrm{DRF} 2}$ is 0.5351 for Fig. 10b. Substituting $f_{\mathrm{DRF} 1}$ and $f_{b 1}$ into Eq. (15) gives $f_{\text {dist }}=f_{b 1}+$ $f_{\mathrm{DRF} 1}=0.6151 \mathrm{~Hz}$, and substituting $f_{\mathrm{DRF} 2}$ and $f_{b 2}$ into Eq. (15) gives $f_{\text {dist }}=f_{b 2}+f_{\text {DRF2 }}=0.6151 \mathrm{~Hz}$.

Note that the first method, evaluating the high-frequency oscillation to determine the input frequency, could have been performed on any of the CIT plots. We would again find the input disturbance frequency to be $0.6151 \mathrm{~Hz}$. Therefore, if the filter designer has an estimate of the actual input disturbance frequency, the designer can use a close DRF rejection frequency to produce beats in the CIT and explicitly determine the actual disturbance frequency. Notice that the simple system identification method can be implemented whether the plant is on-site or remote, such as on an orbiting satellite.

When the two frequency determination methods are compared, there are some advantages to utilizing the method employing beats. Using the proposed beats method, the designer is able to easily visualize if the rejection frequency $\omega_{p}$ is correct or not. If beats appear, then $\omega_{p}$ is incorrect. As the designer alters $\omega_{p}$, either more or less beats will appear. As stated earlier, if $\omega_{p}$ is increased toward the actual disturbance frequency, less beats will occur, and the CIT magnitude will increase to the input disturbance magnitude. ${ }^{12}$ Therefore, by employing the beats to determine the input disturbance frequency, the designer has two simple visual trends that reveal if the rejection frequency is correct: 1) decrease in beats and 2) increase in CIT magnitude. Although not developed further here, these trends could also be automated to tune the filters on-orbit, which would be especially helpful if the disturbance frequency was changing over time.

\section{Conclusions}

This paper investigated spacecraft disturbance rejection for periodic disturbances using the NPS TAS. It successfully demonstrated a dipole DRF, based on the internal model principle. Also, it showed that the filter is not robust to frequency uncertainty. Designing the filter with an error of 5\% in the disturbance frequency essentially rendered the disturbance filter ineffective. As a result, two simple closed-loop system identification methods were introduced to identify experimentally the disturbance frequency to be used within the filter design. One system identification procedure took advantage of the phenomenon that when a faulty frequency is used to design the filter, beating appears in the input control torque. The beat frequency is related to the actual disturbance frequency.

Three natural extensions of this work would make for valuable further work. First, as noted earlier, we could not validate our DRF methods directly on the hardware test bed due to actuator saturation in our reaction wheels. In fact, the needed reaction wheel torque is related to the disturbance magnitude, which saturated our current reaction wheels. A study of the affect of actuator saturation on DRF methods would be useful. In this paper, we considered a flexible beam only as a disturbance source to a rigid body. In fact, the flexibility of the beam could be studied in two ways: 1) Damping in the beam can be accounted for by damping terms in the rejection filter. 2) Possible control-structure interactions could be explored. Also, we noted earlier that our closed-loop methods of frequency identification could be used to create automatic DRF filter tuning onboard orbiting spacecraft. This could be especially helpful if the disturbance frequency were changing over time. The formalization of an integrated DRF, system identification, and system tuning architecture into an online adaptive system would be valuable.

\section{Acknowledgments}

We would like to gratefully acknowledge Bong Wie of Arizona State University for helpful e-mail correspondence. We would also like to thank the associate editor and anonymous reviewers for their valuable comments and suggestions.

\section{References}

${ }^{1}$ Heiberg, C. J., Bailey, D., and Wie, B., "Precision Spacecraft Pointing Using Single-Gimbal Control Moment Gyroscopes with Disturbance," Journal of Guidance, Control, and Dynamics, Vol. 23, No. 1, 2000, pp. $77-85$.

${ }^{2}$ Nurre, G. S., Sharkey, J. P., Nelson, J. D., and Bradley, A. J., "Preservicing Mission, On-Orbit Modifications to Hubble Space Telescope Pointing Control System," Journal of Guidance, Control, and Dynamics, Vol. 18, No. 2, 1995, pp. 222-229.

${ }^{3}$ Agrawal, B. N., "Acquisition, Tracking, and Pointing of Bifocal Relay Mirror Spacecraft," American Astronautical Society, Paper, AAS 03-151, Feb. 2003.

${ }^{4}$ Agrawal, B. N., Romano, M., and Martinez, T., "Three Axis Attitude Control Simulators for Bifocal Relay Mirror Spacecraft," Advances in the Astronautical Sciences, Vol. 115, No. AAS-03-268, 2003.

${ }^{5}$ Chen, C., Linear System Theory and Design, Oxford Univ. Press, New York, 1999, Chap. 8.3.1, pp. 243-247.

${ }^{6}$ Wie, B., and Gonzalez, M., "Control Synthesis for Flexible Space Structures Excited by Persistent Disturbances," Journal of Guidance, Control, and Dynamics, Vol. 15, No. 1, 1992, pp. 73-80.

${ }^{7}$ Wie, B., Space Vehicle Dynamics and Control, AIAA Educational Series, AIAA, Reston, VA, 1998 
${ }^{8}$ Wie, B., Liu, Q., and Bauer, F., "Classical and Robust $\mathrm{H}_{\infty}$ Control Redesign for the Hubble Space Telescope," Journal of Guidance, Control, and Dynamics, Vol. 16, No. 6, 1993, pp. 1069-1077.

${ }^{9}$ Heiberg, C. J., Bailey, D., and Wie, B., "Precision Control Moment Gyroscope Spacecraft Control with Disturbance," Society of Photo-Optical Instrumentation Engineers, Paper 3041-93, March 1997.

${ }^{10}$ Wie, B., Horta, L., and Sulla, J., "Classical Control System Design and Experiment for the Minimast Truss Structure," Journal of Guidance, Control, and Dynamics, Vol. 14, No. 4, 1991, pp. 778-784.

${ }^{11}$ Wie, B., "Experimental Demonstration of a Classical Approach to Flexi- ble Structure Control," Journal of Guidance, Control, and Dynamics, Vol. 15, No. 6, 1992, pp. 1327-1333.

${ }^{12}$ Lau, J., "Spacecraft Attitude Control and Disturbance Rejection," M.S. Thesis, Dept. of Mechanical and Aeronautical Engineering, University of California, Davis, Davis, CA, Dec. 2004.

${ }^{13}$ Bryson, A., Control of Spacecraft and Aircraft, Princeton Univ. Press, Princeton, NJ, 1994.

${ }^{14}$ Wertz, J. (ed.), Spacecraft Attitude Determination and Control, Kluwer Academic, Dordrecht, The Netherlands, 1978.

${ }^{15}$ Serway, R. A., Physics For Scientists \& Engineers, Harcout Brace, Orlando, FL, 1992, pp. 492-495. 